\title{
Third Stokes Parameter Emission from a Periodic Water Surface
}

\author{
J. T. Johnson, J. A. Kong, R. T. Shin, and D. H. Staelin \\ Department of Electrical Engineering and Computer Science \\ and Research Laboratory of Electronics \\ Massachusetts Institute of Technology, Cambridge, MA
}

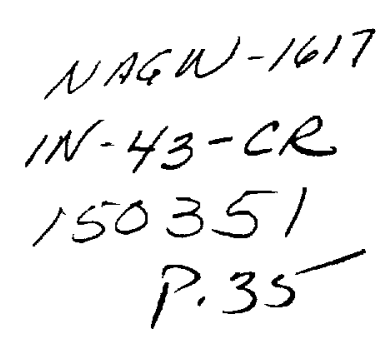

\author{
K. O'Neill and A. Lohanick \\ Cold Regions Research and Engineering Laboratory, Hanover, NH
}

\begin{abstract}
An experiment in which the third Stokes parameter thermal emission from a periodic water surface was measured is documented. This parameter is shown to be related to the direction of periodicity of the periodic surface and to approach brightnesses of up to $30 \mathrm{~K}$ at $X$ band for the surface used in the experiment. The surface actually analyzed was a 'two-layer' periodic surface; the theory of thermal emission from such a surface is derived and the theoretical results are found to be in good agreement with the experimental measurements. These results further the idea of using the third Stokes parameter emission as an indicator of wind direction over the ocean.
\end{abstract}

\section{Introduction}

Recent theoretical works have suggested the potential of passive polarimetry in the remote sensing of geophysical media [1-3]. These works indicate that the third Stokes parameter, $U_{B}$, of the thermal emission may become large for azimuthally asymmetric fields of observation. In [3], values of $U_{B}$ as high as $40 \mathrm{~K}$ were measured from a periodic triangular soil surface at $10 \mathrm{GHz}$. In order to demonstrate the existence of the $U_{B}$ parameter for a water surface, and thus to investigate the potential applicability of passive polarimetry to ocean remote sensing, observations of a periodic water surface were performed at the Cold Regions Research and Engineering Laboratory (CRREL), Hanover, NH in September of 1992. This paper documents those observations and the theory developed for the "two-layer" periodic surface actually analyzed in the experiment. 


\section{Theory of Polarimetry}

In passive polarimetry, brightness temperatures corresponding to all four modified Stokes parameters are measured. The brightness temperature Stokes vector is defined as

$$
\vec{T}_{B}=\frac{1}{C} \bar{I}=\frac{1}{C}\left[\begin{array}{c}
I_{h} \\
I_{v} \\
U \\
V
\end{array}\right]=\frac{1}{\eta C}\left[\begin{array}{c}
\left\langle E_{h} E_{h}^{*}\right\rangle \\
\left\langle E_{v} E_{v}^{*}\right\rangle \\
2 \operatorname{Re}\left\langle E_{v} E_{h}^{*}\right\rangle \\
2 \operatorname{Im}\left\langle E_{v} E_{h}^{*}\right\rangle
\end{array}\right]
$$

In the above equation, $E_{h}$ and $E_{v}$ are the emitted electric fields received from the horizontal and vertical polarization channels of the radiometer, $\eta$ is the characteristic impedance, and $C=K / \lambda^{2}$ with $K$ denoting Boltzmann's constant, $\lambda$ the wavelength. The first two parameters of the brightness temperature Stokes vector correspond to the received powers for horizontal and vertical polarizations, respectively. The third and fourth parameters correspond to the complex correlation between the electric fields received by the horizontal and vertical channels. We label the four parameters $T_{B h}, T_{B v}, U_{B}$, and $V_{B}$ respectively in this paper.

It is shown in [2] that the third and fourth Stokes parameters may be related to the brightness temperature in a 45 degree linearly polarized measurement $\left(T_{B_{p}}\right)$ and a righthand-circularly polarized measurement $\left(T_{B r}\right)$ as follows:

$$
\begin{aligned}
& U_{B}=2 T_{B p}-T_{B h}-T_{B v} \\
& V_{B}=2 T_{B r}-T_{B h}-T_{B v}
\end{aligned}
$$

Thus, to measure all four parameters of the Stokes vector, the brightness temperatures in horizontal, vertical, 45 degree linear, and right-hand-circular polarizations are first measured, and the above equations are used to obtain $U_{B}$ and $V_{B}$.

In the passive remote sensing of rough surfaces, the parameter that is actually of interest is the emissivity, which relates the brightness temperature emitted by an object to its actual physical temperature, under the assumption that the object is at a constant 
physical temperature and that the emission from the object is the only source of brightness:

$$
T_{B a}=e_{a}(\theta, \phi) T_{\text {phys }}
$$

In the above equation, the subscript $a$ refers to the polarization of the brightness temperature, $\theta$ to the polar observation angle, and $\phi$ to the azimuthal observation angle. Through the principles of energy conservation and reciprocity, Kirchhoff's Law relates this emissivity to the reflectivity of the surface [4]:

$$
e_{a}(\theta, \phi)=1-r_{a}(\theta, \phi)
$$

The reflectivity $r_{a}(\theta, \phi)$ for the given incident polarization $a$ is defined as the fraction of the power incident from direction $(\theta, \phi)$ that is rescattered and can be evaluated by integrating the bistatic scattering coefficients $\gamma_{b a}\left(\theta, \phi ; \theta^{\prime}, \phi^{\prime}\right)$ over all scattering angles in the upper hemisphere and summing the results of both orthogonal scattering polarizations.

$$
r_{a}(\theta, \phi)=\frac{1}{4 \pi} \sum_{b} \int_{0}^{\pi / 2} d \theta^{\prime} \sin \theta^{\prime} \int_{0}^{2 \pi} d \phi^{\prime} \gamma_{b a}\left(\theta, \phi ; \theta^{\prime}, \phi^{\prime}\right)
$$

In the expression of the bistatic scattering coefficient, $(\theta, \phi)$ and $\left(\theta^{\prime}, \phi^{\prime}\right)$ represent the incident and the scattered directions, respectively, and the subscripts $a$ and $b$ represent the polarizations of the incident and the scattered waves, respectively.

Thus, to calculate the fully polarimetric emission vector, the bistatic scattering coefficient for each of four polarizations is first calculated and integrated over the upper hemisphere to obtain the reflectivity for that particular polarization. Multiplication of the corresponding emissivity by the physical temperature of the object under view yields the brightness temperature for this polarization. The fully polarimetric brightness vector is then calculated as described previously.

\section{Two-Layer Periodic Surface Theory}

In order to form a periodic water surface, a thin sinusoidal sheet of fiberglass was placed on top of a flat water surface. After air bubbles trapped underneath were removed, a 
"two-layer" periodic surface resulted. To examine the effects of this layer on the measured brightness temperatures, a theoretical model for thermal emission from a two-layer periodic surface was derived and implemented.

The problem of calculating thermal emission from a "single-layer" periodic surface has been solved by several researchers using many different techniques. Two of the methods used in the single-layer case are extended for the two-layer case in this analysis. The results from these two 'exact' methods are then compared to determine which would apply best to the cases measured in the experiment. For both of these methods, the calculations are performed to determine the total power reflected in the reciprocal active problem; this total reflectivity is then used to obtain the emissivity as described previously.

The extended boundary condition (EBC) method described by Chuang and Kong [5] for calculating scattering from a single-layer periodic surface is the first method to be applied. In this method, Huygens' principle is applied at the surface so that the scattered field can be obtained once the surface fields are known. The Huygens' integral equation is solved by expanding the unknown surface fields into Fourier series and forming a truncated impedance matrix which is inverted to obtain the surface fields. This method is known as the extended boundary condition method because the calculations enforce the requirement that the Huygens' integral which equals the field value within the region of interest must be zero outside that region. Although the extended boundary condition method is exact, the numerical formulation becomes ill conditioned if the surface height-to-period ratio becomes too large [10].

To overcome this problem, a second method, the method of moments [9], can be used to perform the calculations. In this formulation, the same Huygens' principle equation is used, but the requirement that the Huygens' integral in the region not of interest be zero is not enforced. Instead, the integral equation is "tested" at a discrete number of points along each surface where the equation is forced to hold. As the number of testing points is increased, the calculated scattered fields converge to the exact results. The method of 
moments can be used in cases where the extended boundary condition method fails. It requires, however, more computation time than the EBC.

\subsection{General Formulation}

Consider a plane wave incident upon a two-layer periodic surface described by $f_{1}(x)=$ $f_{1}(x+P), f_{2}(x)=f_{2}(x+P)$ with $f_{1}$ representing the upper surface, $f_{2}$ the lower surface and $P$ denoting the period of the surface in the $\hat{x}$ direction (see figure 1 ). The electric field of the incident wave is given by

$$
\bar{E}_{i}=\hat{e}_{i} E_{0} e^{i \bar{k}_{i} \cdot \bar{F}}
$$

where $\bar{k}_{i}$ denotes the incident wave vector and is equal to $\hat{x} k_{x i}+\hat{y} k_{y i}-\hat{z} k_{z i}$ and $\hat{e}_{i}$ is the polarization of the electric field vector.

Since the structure is uniform in the $\hat{y}$ direction, all the field components in regions 0,1 , and 2 will be phase matched to the same $\exp \left(i k_{y i} y\right)$ dependence. Thus, we can replace all the $\frac{\partial}{\partial y}$ terms in Maxwell's equations with $i k_{y i}$ as is commonly done in waveguide theory (usually with the $z$ dependence by convention.) Subsequent equations will thus have the $\exp \left(i k_{y i} y\right)$ dependence removed.

Maxwell's equations can now be simplified so that the $\hat{x}$ and $\hat{z}$ components of the electric and magnetic fields can be expressed as functions of the $\hat{y}$ components of the fields as follows:

$$
\begin{aligned}
& \bar{E}_{j \diamond}(\bar{r})=\frac{i}{k_{j}{ }^{2}-k_{y i}{ }^{2}}\left[k_{y i} \nabla, E_{j y}(\bar{r})+\omega \mu \nabla, \times \bar{H}_{j} y(\bar{r})\right] \\
& \bar{H}_{j \diamond}(\bar{r})=\frac{i}{k_{j}{ }^{2}-k_{y i}{ }^{2}}\left[k_{y i} \nabla_{\iota} H_{j y}(\bar{r})-\omega \epsilon \nabla, \times \bar{E}_{j} y(\bar{r})\right]
\end{aligned}
$$

where $j=0,1,2$ signifies regions 0,1 , or 2 , respectively, $\nabla$, is the gradient operator that is transverse to the $\hat{y}$ direction

$$
\nabla_{.}=\hat{x} \frac{\partial}{\partial x}+\hat{z} \frac{\partial}{\partial z}
$$

and $s=(x, z)$ indicates the transverse components of the fields for region $j$. The $\hat{y}$ 
components of the electric and magnetic fields now satisfy the partial differential equations

$$
\left(\nabla^{2}+k_{j}{ }^{2}-k_{y i}{ }^{2}\right) A_{j y}=0
$$

where $A_{j y}=E_{j y}, H_{j y}$. Since $\nabla$, operates only in a two-dimensional space, the $\hat{y}$ components of the electric and magnetic fields satisfy a two-dimensional wave equation. The Green's function for such an equation is given by

$$
\begin{gathered}
G_{j}\left(\bar{\rho}_{s}, \bar{\rho}_{s}^{\prime}\right)=\frac{i}{4} H_{0}^{(1)}\left(k_{j s}\left|\bar{\rho}_{s}-\bar{\rho}_{s}^{\prime}\right|\right) \\
=\frac{i}{4 \pi} \int_{-\infty}^{\infty} d k_{x} \frac{1}{k_{j z}} \times \exp \left[i k_{x}\left(x-x^{\prime}\right)+i k_{j z}\left|z-z^{\prime}\right|\right]
\end{gathered}
$$

where $j=0,1,2$,

$$
\begin{gathered}
k_{j,}=\left(k_{j}{ }^{2}-k_{y i}{ }^{2}\right)^{1 / 2} \\
k_{j x}=\sqrt{k_{j,}^{2}-k_{x}^{2}}
\end{gathered}
$$

and

$$
\bar{\rho}_{,}=\hat{x} x+\hat{z} z
$$

Applying the scalar Green's function form of Huygens' principle to the top periodic surface and region 0 gives

$$
\begin{aligned}
& E_{y i}\left(\bar{\rho}_{s}\right)-\int_{-\infty}^{\infty} d \sigma^{\prime}\left\{G_{0}\left(\bar{\rho}_{s}, \bar{\rho}_{s}^{\prime}\right) \hat{n}_{1} \cdot \nabla_{s}^{\prime} E_{0 y}\left(\bar{\rho}_{s}^{\prime}\right)-E_{0 y}\left({ }_{s}^{\prime}\right) \hat{n}_{1} \cdot \nabla_{s}^{\prime} G_{0}\left(\bar{\rho}_{s}, \bar{\rho}_{s}^{\prime}\right)\right\} \\
& = \begin{cases}E_{0 y}\left(\bar{\rho}_{s}\right) & z>f_{1}(x) \\
0 & z<f_{1}(x)\end{cases}
\end{aligned}
$$

where the $\hat{y}$ dependence has been suppressed and

$$
d \sigma^{\prime} \hat{n}_{j}=\left[\hat{z}-\hat{x} \frac{d f_{j}\left(x^{\prime}\right)}{d x^{\prime}}\right] d x^{\prime}
$$

The above surface integral is over an infinite domain. However, the periodic properties of the surface fields can be used to reduce the integration domain to a single period. The surface fields have the property

$$
\omega\left(\bar{\rho}_{s}+\hat{x} n P\right)=\omega\left(\bar{\rho}_{s}\right) e^{i k_{\varepsilon i} n P}
$$


where $\omega\left(\bar{\rho}_{s}\right)$ can be $E_{j y}\left(\bar{\rho}_{s}\right), H_{j y}\left(\bar{\rho}_{s}\right)$ or their normal derivatives, $n$ is an integer, and $P$ is the period of the surface.

Following Chuang and Kong [5], a periodic Green's function can be derived using this property. This Green's function is

$$
G_{j P}\left(\bar{\rho}_{s}, \bar{\rho}_{\iota}^{\prime}\right)=\frac{-1}{2 i k_{j s} P} \sum_{n} \frac{1}{\beta_{j n}} \exp \left[i k_{j \_} \alpha_{j n}\left(x-x^{\prime}\right)+i k_{\lrcorner} \beta_{j n}\left|z-z^{\prime}\right|\right]
$$

where

$$
\begin{gathered}
\alpha_{j n}=\frac{k_{x i}+n \frac{2 \pi}{P}}{k_{j s}} \\
\beta_{j n}= \begin{cases}\left(1-\alpha_{j n}^{2}\right)^{1 / 2} & \alpha_{j n}^{2}<1 \\
i\left(1-\alpha_{j n}^{2}\right)^{1 / 2} & \alpha_{j n}^{2}>1\end{cases}
\end{gathered}
$$

Now using the periodic Green's function in the scalar Huygens' principle formulation for each of the three regions yields

Region zero

$$
\begin{aligned}
& E_{y i}\left(\bar{\rho}_{s}\right)-\int_{P(S 1)} d \sigma^{\prime}\left\{G_{0 P}\left(\bar{\rho}_{s}, \bar{\rho}_{s}^{\prime}\right) \hat{n}_{1} \cdot \nabla_{s}^{\prime} E_{0 y}\left(\bar{\rho}_{s}^{\prime}\right)-E_{0 y}\left(\bar{\rho}_{s}^{\prime}\right) \hat{n}_{1} \cdot \nabla_{s}^{\prime} G_{0 P}\left(\bar{\rho}_{s}, \vec{\rho}_{s}^{\prime}\right)\right\} \\
& = \begin{cases}E_{0 y}\left(\bar{\rho}_{s}\right) & z>f_{1}(x) \\
0 & z<f_{1}(x)\end{cases} \\
& H_{y i}\left(\bar{\rho}_{s}\right)-\int_{P(S 1)} d \sigma^{\prime}\left\{G_{0 P}\left(\bar{\rho}_{s}, \bar{\rho}_{s}^{\prime}\right) \hat{n}_{1} \cdot \nabla_{s}^{\prime} H_{\mathrm{O}_{y}}\left(\bar{\rho}_{s}^{\prime}\right)-H_{\mathrm{Oy}}\left(\bar{\rho}_{s}^{\prime}\right) \hat{n}_{1} \cdot \nabla_{s}^{\prime} G_{0 P}\left(\bar{\rho}_{s}, \bar{\rho}_{s}^{\prime}\right)\right\} \\
& = \begin{cases}H_{0_{y}}\left(\bar{\rho}_{a}\right) & z>f_{1}(x) \\
0 & z<f_{1}(x)\end{cases}
\end{aligned}
$$

Region one

$$
\begin{array}{r}
\int_{P(S 1)} d \sigma^{\prime}\left\{G_{1 P}\left(\bar{\rho}_{s}, \bar{\rho}_{s}^{\prime}\right) \hat{n}_{1} \cdot \nabla_{s}^{\prime} E_{1 y}\left(\bar{\rho}_{s}^{\prime}\right)-E_{1 y}\left(\bar{\rho}_{s}^{\prime}\right) \hat{n}_{1} \cdot \nabla_{s}^{\prime} G_{1 P}\left(\bar{\rho}_{s}, \bar{\rho}_{s}^{\prime}\right)\right\}- \\
\int_{P(S 2)} d \sigma^{\prime}\left\{G_{1 P}\left(\bar{\rho}_{s}, \bar{\rho}_{s}^{\prime}\right) \hat{n}_{2} \cdot \nabla_{s}^{\prime} e_{1 y}\left(\bar{\rho}_{s}^{\prime}\right)-E_{1 y}\left(\bar{\rho}_{s}^{\prime}\right) \hat{n}_{2} \cdot \nabla_{s}^{\prime} G_{1 P}\left(\bar{\rho}_{s}, \bar{\rho}_{s}^{\prime}\right)\right\} \\
= \begin{cases}0 & z>f_{1}(x) \\
E_{1 y}\left(\bar{\rho}_{s}\right) & f_{2}(x)<z<f_{1}(x) \\
0 & z<f_{2}(x)\end{cases}
\end{array}
$$




$$
\begin{array}{r}
\int_{P(S 1)} d \sigma^{\prime}\left\{G_{1 P}\left(\bar{\rho}_{s}, \bar{\rho}_{s}^{\prime}\right) \hat{n}_{1} \cdot \nabla_{s}^{\prime} H_{1 y}\left(\bar{\rho}_{s}^{\prime}\right)-H_{1 y}\left(\bar{\rho}_{s}^{\prime}\right) \hat{n}_{1} \cdot \nabla_{s}^{\prime} G_{1 P}\left(\bar{\rho}_{s}, \bar{\rho}_{s}^{\prime}\right)\right\}- \\
\int_{P(S 2)} d \sigma^{\prime}\left\{G_{1 P}\left(\bar{\rho}_{s}, \vec{\rho}_{s}^{\prime}\right) \hat{n}_{2} \cdot \nabla_{s}^{\prime}, H_{1 y}\left(\bar{\rho}_{s}^{\prime}\right)-H_{1 y}\left(\bar{\rho}_{s}^{\prime}\right) \hat{n}_{2} \cdot \nabla_{s}^{\prime} G_{1 P}\left(\bar{\rho}_{s}, \bar{\rho}_{s}^{\prime}\right)\right\} \\
= \begin{cases}0 & z>f_{1}(x) \\
H_{1 y}\left(\bar{\rho}_{s}\right) & f_{2}(x)<z<f_{1}(x) \\
0 & z<f_{2}(x)\end{cases}
\end{array}
$$

Region two

$$
\begin{aligned}
& \int_{P(S 2)} d \sigma^{\prime}\left\{G_{2 P}\left(\bar{\rho}_{s}, \bar{\rho}_{s}^{\prime}\right) \hat{n}_{2} \cdot \nabla_{s}^{\prime} E_{2 y}\left(\bar{\rho}_{s}^{\prime}\right)-E_{2 y}\left(\bar{\rho}_{s}^{\prime}\right) \hat{n}_{2} \cdot \nabla_{s}^{\prime} G_{2 P}\left(\bar{\rho}_{s}, \bar{\rho}_{s}^{\prime}\right)\right\} \\
& = \begin{cases}E_{2 y}\left(\bar{\rho}_{s}\right) & z<f_{2}(x) \\
0 & z>f_{2}(x)\end{cases} \\
& \int_{P(S 2)} d \sigma^{\prime}\left\{G_{2 P}\left(\bar{\rho}_{s}, \bar{\rho}_{s}^{\prime}\right) \hat{n}_{2} \cdot \nabla_{a}^{\prime} H_{2 y}\left(\bar{\rho}_{s}^{\prime}\right)-H_{2 y}\left(\bar{\rho}_{s}^{\prime}\right) \hat{n}_{2} \cdot \nabla_{s}^{\prime} G_{2 P}\left(\bar{\rho}_{s}, \bar{\rho}_{s}^{\prime}\right)\right\} \\
& = \begin{cases}H_{2 y}\left(\bar{\rho}_{s}\right) & z<f_{2}(x) \\
0 & z>f_{2}(x)\end{cases}
\end{aligned}
$$

where $(S l)$ indicates that the integration is performed over surface $l$.

Once the surface fields are determined, the fields in regions 0,1 , and 2 can be obtained using these relations. At present, there are sixteen unknown vectors to be obtained: the $\hat{y}$ components of the electric and magnetic fields at surface one for regions zero and one, the

$\hat{y}$ components of the electric and magnetic fields at surface two for regions one and two, and the normal derivatives of the $\hat{y}$ component of the electric and magnetic fields for each of these surface-region combinations. Electric and magnetic field boundary conditions can be used at both surfaces to reduce the number of unknown vectors to eight.

\subsection{Boundary Conditions}

At both surfaces, the $\hat{y}$ component of the electric and magnetic fields are tangential, and therefore must be continuous: 


$$
\begin{aligned}
& E_{0 y}=E_{1 y} \quad z=f_{1}(x) \\
& H_{0 y}=H_{1 y} \\
& E_{1 y}=E_{2 y} \quad z=f_{2}(x) \\
& H_{1 y}=H_{2 y}
\end{aligned}
$$

The components of the fields along the surface profile are also tangential and must be continuous. These relations can be written as:

$$
\begin{aligned}
& \hat{n}_{1} \times \bar{E}_{0,}=\hat{n}_{1} \times \bar{E}_{1 \triangleleft}, z=f_{1}(x) \\
& \hat{n}_{1} \times \bar{H}_{0 \iota}=\hat{n}_{1} \times \bar{H}_{1 \triangleleft} \\
& \hat{n}_{2} \times \bar{E}_{1 \triangleleft}=\hat{n}_{2} \times \bar{E}_{2,}, z=f_{2}(x) \\
& \hat{n}_{2} \times \bar{H}_{1 \triangleleft}=\hat{n}_{2} \times \bar{H}_{2 \triangleleft}
\end{aligned}
$$

Note that both the normal and the $s$ component fields lie in the $x-z$ plane, so that $\hat{n}_{j} \times \bar{A}$, is a $\hat{y}$ directed vector whose amplitude corresponds to the tangential component of the field along the surface profile. Following Chuang and Kong [5], the second set of boundary conditions can be written as:

$$
\begin{gathered}
\hat{y}\left(\hat{n}_{1} \cdot \nabla \cdot E_{0 y}\right)=c_{0} \hat{n}_{1} \times \nabla \cdot H_{1 y}+c_{2} \hat{y}\left(\hat{n}_{1} \cdot \nabla, E_{1 y}\right) \quad z=f_{1}(x) \\
\hat{y}\left(\hat{n}_{1} \cdot \nabla, H_{0 y}\right)=-d_{0} \hat{n}_{1} \times \nabla, E_{1 y}+d_{2} \hat{y}\left(\hat{n}_{1} \cdot \nabla, H_{1 y}\right) \\
\hat{y}\left(\hat{n}_{2} \cdot \nabla, E_{1 y}\right)=c_{1} \hat{n}_{2} \times \nabla, H_{2 y}+c_{3} \hat{y}\left(\hat{n}_{2} \cdot \nabla, E_{2 y}\right) \quad z=f_{2}(x) \\
\hat{y}\left(\hat{n}_{2} \cdot \nabla, H_{1 y}\right)=-d_{1} \hat{n}_{2} \times \nabla_{\imath} E_{2 y}+d_{3} \hat{y}\left(\hat{n}_{2} \cdot \nabla, H_{2 y}\right)
\end{gathered}
$$

where

$$
c_{0}=\left[\frac{k_{0 s}^{2}}{k_{1 s}^{2}}-1\right] \frac{k_{y i}}{\omega \epsilon_{0}} \quad c_{1}=\left[\frac{k_{1 s}^{2}}{k_{2 s}^{2}}-1\right] \frac{k_{y i}}{\omega \epsilon_{1}}
$$




$$
\begin{aligned}
c_{2}=\frac{\epsilon_{1}}{\epsilon_{0}} \frac{k_{0 s}^{2}}{k_{1 s}^{2}} & c_{3}=\frac{\epsilon_{2}}{\epsilon_{1}} \frac{k_{1 s}^{2}}{k_{2 s}^{2}} \\
d_{0}=\left[\frac{k_{0 s}^{2}}{k_{1 s}^{2}}-1\right] \frac{k_{y i}}{\omega \mu_{0}} & d_{1}=\left[\frac{k_{1 s}^{2}}{k_{2 s}^{2}}-1\right] \frac{k_{y i}}{\omega \mu_{1}} \\
d_{2}=\frac{\mu_{1}}{\mu_{0}} \frac{k_{0 s}^{2}}{k_{1 s}^{2}} & d_{3}=\frac{\mu_{2}}{\mu_{1}} \frac{k_{1 s}^{2}}{k_{2 s}^{2}}
\end{aligned}
$$

These eight boundary conditions reduce the sixteen unknown vectors to eight. The remaining eight unknowns are then solved by expanding the surface fields in the Huygens' integral equations into a sum of unknown amplitude basis functions. The functions used in this expansion and the equations solved differ for the EBC and Moment methods; each method will be treated separately beginning with the EBC.

\subsection{Extended Boundary Condition Method}

If we substitute the expression for the periodic Green's function into the Huygens' Principle integral equations and factor out the unprimed exponential dependence, the equations reduce to:

Region zero

$$
\begin{aligned}
E_{0 y}\left(\bar{\rho}_{s}\right) & =E_{y i}+\sum_{n} b_{01 n} \frac{e^{i k_{0 n}^{+} \cdot \bar{\rho}_{s}}}{\sqrt{\beta_{0 n}}} & & z>f_{1(\max )} \\
0 & =E_{y i}-\sum_{n} a_{01 n} \frac{e^{i \hat{k}_{0 n}^{-} \cdot \rho_{s}}}{\sqrt{\beta_{0 n}}} & & z<f_{1(\min )} \\
H_{0 y}\left(\bar{\rho}_{s}\right) & =H_{y i}+\sum_{n} b_{01 n}^{(h)} \frac{e^{i k_{0 n}^{+} \cdot \beta_{s}}}{\sqrt{\beta_{0 n}}} & & z>f_{1(\max )} \\
0 & =H_{y i}-\sum_{n} a_{01 n}^{(h)} \frac{e^{i k_{0 n}^{-} \cdot \rho_{s}}}{\sqrt{\beta_{0 n}}} & & z<f_{1(\min )}
\end{aligned}
$$

Region one

$$
\begin{aligned}
0 & =\sum_{n} b_{11 n} \frac{e^{i k_{1 n}^{+} \cdot \bar{\beta}_{s}}}{\sqrt{\beta_{1 n}}}+\sum_{n} b_{12 n} \frac{e^{i k_{1 n}^{+} \cdot \bar{\rho}_{s}}}{\sqrt{\beta_{1 n}}} \quad z>f_{1(\max )} \\
E_{1 y}\left(\bar{\rho}_{\iota}\right) & =\sum_{n} b_{12 n} \frac{e^{i k_{1 n}^{+} \cdot \bar{\rho}_{s}}}{\sqrt{\beta_{1 n}}}+\sum_{n} a_{11 n} \frac{e^{i k_{1 n}^{-} \cdot \bar{p}_{s}}}{\sqrt{\beta_{1 n}}} \\
f_{2(\max )} & <z<f_{1(\min )}
\end{aligned}
$$




$$
\begin{aligned}
0 & =\sum_{n} a_{11 n} \frac{e^{i k_{1 n}^{-} \cdot \bar{\rho}_{s}}}{\sqrt{\beta_{1 n}}}+\sum_{n} a_{12 n} \frac{e^{i \bar{k}_{1 n}^{-} \cdot \bar{\rho}_{1}}}{\sqrt{\beta_{1 n}}} \quad z<f_{2(\min )} \\
0 & =\sum_{n} b_{11 n}^{(h)} \frac{e^{i \bar{k}_{1 n}^{+} \cdot \rho_{s}}}{\sqrt{\beta_{1 n}}}+\sum_{n} b_{12 n}^{(h)} \frac{e^{i k_{1 n}^{+} \cdot \bar{\rho}_{s}}}{\sqrt{\beta_{1 n}}} \quad z>f_{1(\max )} \\
H_{1 y}\left(\bar{\rho}_{s}\right) & =\sum_{n} b_{12 n}^{(h)} \frac{e^{i k_{1 n}^{+} \cdot \bar{\rho}_{s}}}{\sqrt{\beta_{1 n}}}+\sum_{n} a_{11 n}^{(h)} \frac{e^{i \bar{k}_{1 n}^{-} \cdot \bar{\rho}_{s}}}{\sqrt{\beta_{1 n}}} \\
0 & =\sum_{n} a_{11 n}^{(h)} \frac{e^{i \bar{k}_{1 n}^{-} \cdot \bar{\rho}_{s}}}{\sqrt{\beta_{1 n}}}+\sum_{n} a_{12 n}^{(h)} \frac{e^{i \overline{1}_{1 n}^{-} \cdot \bar{\rho}_{s}}}{\sqrt{\beta_{1 n}}} \quad z<f_{2(\min )}
\end{aligned}
$$

\section{Region two}

$$
\begin{aligned}
E_{2 y}\left(\bar{\rho}_{s}\right) & =\sum_{n} b_{22 n} \frac{e^{i k_{2 n}^{+} \cdot \bar{\rho}_{d}}}{\sqrt{\beta_{2 n}}} \quad z<f_{2(\min )} \\
0 & =-\sum_{n} a_{22 n} \frac{e^{i k_{2 n}^{-} \cdot \rho_{s}}}{\sqrt{\beta_{0 n}}} \quad z>f_{2(\max )} \\
H_{2 y}\left(\bar{\rho}_{s}\right) & =\sum_{n} b_{22 n}^{(h)} \frac{e^{i k_{2 n}^{+} \cdot \rho_{s}}}{\sqrt{\beta_{2 n}}} \quad z<f_{2(\min )} \\
0 & =-\sum_{n} a_{22 n}^{(h)} \frac{e^{i \bar{k}_{2 n}^{-} \cdot \bar{\rho}_{s}}}{\sqrt{\beta_{2 n}}} \quad z>f_{2(\max )}
\end{aligned}
$$

where the $\left|z-z^{\prime}\right|$ expression in the periodic Green's function has been replaced by $z-z^{\prime}$ when the observation point is above the surface, $z^{\prime}-z$ when the observation point is below the surface. In the above equations,

$$
\begin{aligned}
& \bar{k}_{j n}^{ \pm}=\hat{x} k_{j,} \alpha_{j n} \pm \hat{z} k_{j,} \beta_{j n} \\
& b_{j l n}=\frac{1}{2 i k_{j,} P} \int_{P(S l)} d \sigma^{\prime}\left\{\frac{e^{-i k_{j n}^{+} \cdot \bar{\rho}_{\bullet}\left(x^{\prime}\right)}}{\sqrt{\beta_{j n}}} \hat{n}_{l} \cdot \nabla_{\cdot}^{\prime} E_{j y}\left(\vec{\rho}_{\bullet}^{\prime}\right)\right. \\
& \left.-E_{j y}\left(\bar{\rho}_{\bullet}^{\prime}\right) \hat{n}_{l} \cdot \nabla^{\prime} \frac{e^{-i k_{j n}^{+} \cdot \bar{\rho}_{o}\left(x^{\prime}\right)}}{\sqrt{\beta_{j n}}}\right\}
\end{aligned}
$$




$$
\begin{gathered}
a_{j l n}=\frac{-1}{2 i k_{j s} P} \int_{P(S l)} d \sigma^{\prime}\left\{\frac{e^{-i \bar{k}_{j n}^{-} \cdot \bar{\rho}_{s}\left(x^{\prime}\right)}}{\sqrt{\beta_{j n}}} \hat{n}_{l} \cdot \nabla_{s}^{\prime} E_{j y}\left(\bar{\rho}_{s}^{\prime}\right)\right. \\
\left.-E_{j y}\left(\bar{\rho}_{s}^{\prime}\right) \hat{n}_{l} \cdot \nabla^{\prime} \frac{e^{-i \bar{k}_{j n}^{-} \cdot \bar{\rho}_{s}\left(x^{\prime}\right)}}{\sqrt{\beta_{j n}}}\right\}
\end{gathered}
$$

and $b_{j l n}^{(h)}$ and $a_{j l n}^{(h)}$ have the same form as their electric field counterparts with $E_{j y}$ replaced by $H_{j y}$. We now recognize that the scattered field in region 0 is composed of a discrete set of plane waves traveling in the directions indicated by $\bar{k}_{0 n}^{+}$. These plane waves are known as the Floquet modes; the amplitude of the $n$th reflected mode is determined by $b_{01 n}$.

For the extended boundary condition method, we use the equations which involve the region of no interest for each region. These are equations $(46),(48),(49),(51),(52),(54),(56)$, and (58). These eight equations will uniquely determine the eight unknown surface field vectors. Once the surface fields are determined, the remaining equations are used to calculate the scattered field in each of the three regions.

Following Chuang and Kong [5], a Fourier series expansion is used for each of the unknown field quantities as follows:

Surface one

$$
\begin{gathered}
E_{1 y}\left[\bar{\rho}_{\triangleleft}(x)\right]=\sum_{n} 2 \alpha_{n}^{T} \exp \left[i k_{x i} x+i \frac{2 \pi n}{P} x\right] \\
d \sigma \hat{n}_{1} \cdot \nabla, E_{1 y}\left[\bar{\rho}_{\diamond}(x)\right]=i k_{1} d x \sum_{n} 2 \beta_{n}^{T} \exp \left[i k_{x i} x+i \frac{2 \pi n}{P} x\right] \\
H_{1 y}\left[\bar{\rho}_{\triangleleft}(x)\right]=\sum_{n} 2 \gamma_{n}^{T} \exp \left[i k_{x i} x+i \frac{2 \pi n}{P} x\right] \\
d \sigma \hat{n}_{1} \cdot \nabla . H_{1 y}\left[\bar{\rho}_{\triangleleft}(x)\right]=i k_{1} d x \sum_{n} 2 \delta_{n}^{T} \exp \left[i k_{x i} x+i \frac{2 \pi n}{P} x\right]
\end{gathered}
$$

Surface two

$$
\begin{gathered}
E_{2 y}\left[\bar{\rho}_{\triangleleft}(x)\right]=\sum_{n} 2 \alpha_{n}^{B} \exp \left[i k_{x i} x+i \frac{2 \pi n}{P} x\right] \\
d \sigma \hat{n}_{2} \cdot \nabla_{\iota} E_{2 y}\left[\bar{\rho}_{\lrcorner}(x)\right]=i k_{2} d x \sum_{n} 2 \beta_{n}^{B} \exp \left[i k_{x i} x+i \frac{2 \pi n}{P} x\right] \\
H_{2 y}\left[\bar{\rho}_{\lrcorner}(x)\right]=\sum_{n} 2 \gamma_{n}^{B} \exp \left[i k_{x i} x+i \frac{2 \pi n}{P} x\right]
\end{gathered}
$$




$$
\dot{d} \sigma \hat{n}_{2} \cdot \nabla_{s} H_{2 y}\left[\bar{\rho}_{s}(x)\right]=i k_{2 s} d x \sum_{n} 2 \delta_{n}^{B} \exp \left[i k_{x i} x+i \frac{2 \pi n}{P} x\right]
$$

Substituting the above expressions into the eight equations and using the property

$$
d \sigma \hat{n}_{j} \times \nabla_{.} H_{j y}(x, z(x))=\hat{y} d x\left(\frac{\partial H_{j y}}{\partial x}+\frac{d z}{d x} \frac{\partial H_{j y}}{\partial z}\right)=\hat{y} d x\left(\frac{d H_{j y}}{d x}\right)
$$

leads to the coupled matrix equation of Appendix A. Truncating the Fourier series of the surface field unknowns results in a finite matrix equation which can be solved to obtain the surface field unknowns. The upward going field amplitudes are then calculated to be

$$
\begin{gathered}
\bar{b}_{01}=-c_{0} \overline{\bar{C}}_{1}^{+} \gamma^{T}-c_{2} \frac{k_{1 s}}{k_{0 s}} \bar{B}_{1}^{+} \beta^{T}-\overline{\bar{A}}_{1}^{+} \alpha^{T} \\
\bar{b}_{01}^{(h)}=d_{0} \overline{\bar{C}}_{1}^{+} \alpha^{T}-d_{2} \frac{k_{1 s} s}{k_{0 s}} \overline{\bar{B}}_{1}^{+} \delta^{T}-\overline{\bar{A}}_{1}^{+} \gamma^{T}
\end{gathered}
$$

and the downward going field amplitudes in region 2 are

$$
\begin{aligned}
& \bar{a}_{22}=\overline{\bar{E}}_{2}^{-} \beta^{B}+\overline{\bar{D}}_{2}^{-} \alpha^{B} \\
& \bar{a}_{22}^{(h)}=\overline{\bar{E}}_{2}^{-} \delta^{B}+\overline{\bar{D}}_{2}^{-} \gamma^{B}
\end{aligned}
$$

The matrices in the above equations are as described in Appendix A. The above coefficients are the amplitudes of the $\hat{y}$ components of the scattered and transmitted electric and magnetic fields. The $\hat{x}$ and $\hat{z}$ components can be calculated from equations (8) and (9). The scattered plane waves in region zero propagate along the directions determined by $\bar{k}_{0 n}^{+}$, while the transmitted plane waves in region two travel along the directions of $\bar{k}_{2 n}$.

Once the amplitudes of the scattered modes are calculated, the emissivity of the surface can be obtained by integrating the total power reflected over the upper hemisphere and then applying Kirchhoff's law to obtain the emissivity corresponding to the polarization of the incident field. For the $n^{\text {th }}$ propagating scattered Floquet mode, the reflectivity is given by power transmitted in the $\hat{z}$ direction divided by the incident power and is found in [5] to be:

$$
r_{n}=\frac{\left|b_{01 n}\right|^{2}+\left|\eta_{0} b_{01 n}^{(h)}\right|^{2}}{\left|a_{010}\right|^{2}+\left|\eta_{0} a_{010}^{(h)}\right|^{2}}
$$


where the $a_{010}$ and $a_{010}^{(h)}$ are as defined in Appendix A. The brightness temperature of the surface is then given by

$$
T_{B a}=T_{p h y s}\left(1-\sum_{n} r_{n}\right)
$$

where the sum is over the propagating modes reflected for an incident wave polarized in the $a$ direction.

\subsection{Method of Moments}

A second method for solving the Huygens' integral equations for a two-layer periodic surface is the method of moments. The use of the method of moments for computing scattering from a single-layer periodic surface has been presented by Veysoglu et al [2]; these methods will be extended for the two-layer periodic surface in this section.

We begin with the integral equations presented in the general formulation section and the periodic Green's function. We will also use a second form of the periodic Green's function in the calculations

$$
G_{j P}\left(\bar{\rho}, \bar{\rho}^{\prime}\right)=\frac{i}{4} \sum_{m=-\infty}^{\infty} H_{0}^{(1)}\left(k_{j} \sqrt{\left.\left[x-\left(x^{\prime}+m p\right)\right]^{2}+\left(z-z^{\prime}\right)^{2}\right)} \exp \left(i k_{0 x} m p\right)\right.
$$

in addition to the sum of exponentials form used in the EBC method. The eight surface field unknowns in the integral equations are now expanded into sums of unknown amplitude "pulse" functions as follows:

Surface one

$$
\begin{aligned}
E_{1 y}(\bar{r}) & =\sum_{n} a_{n} P_{n}(\bar{r}) \\
\hat{n}_{1} \cdot \nabla, E_{1 y}(\bar{r}) & =\sum_{n} b_{n} P_{n}(\bar{r}) \\
H_{1 y}(\bar{r}) & =\sum_{n} c_{n} P_{n}(\bar{r}) \\
\hat{n}_{1} \cdot \nabla \cdot H_{1 y}(\bar{r}) & =\sum_{n} d_{n} P_{n}(\bar{r})
\end{aligned}
$$

Surface two

$$
E_{2 y}(\bar{r})=\sum_{n} e_{n} P_{n}(\bar{r})
$$




$$
\begin{aligned}
\hat{n}_{2} \cdot \nabla_{\iota} E_{2 y}(\bar{r}) & =\sum_{n} f_{n} P_{n}(\bar{r}) \\
H_{2 y}(\bar{r}) & =\sum_{n} g_{n} P_{n}(\bar{r}) \\
\hat{n}_{2} \cdot \nabla, H_{2 y}(\bar{r}) & =\sum_{n} h_{n} P_{n}(\bar{r})
\end{aligned}
$$

where

$$
P_{n}(\bar{r})=\left\{\begin{array}{lc}
1 & \frac{(n-1) P}{N}<x<\frac{n P}{N} \\
0 & \text { otherwise }
\end{array}\right.
$$

and $N$ is the total number of basis (pulse) functions used.

Instead of solving the equations with right-hand-sides of zero, as was done in the EBC method, the method of moments uses the equations with right-hand-sides corresponding to the fields in the region of interest. These six equations are "tested" at a discrete set of points along the surface upon which the integral is performed where the integral equations are forced to hold. The missing two equations needed to match the eight unknown functions are obtained from testing the integral equations for region one on both surfaces one and two. The testing points are chosen to lie in the center of the pulse basis functions described above. The integral equations now reduce to the matrix equation given in the Appendix B.

Note that this method will require the integration of the singularity in the periodic Green's function when the testing point and integration range overlap. For these "self" terms of the matrices, an asymptotic expression for the Hankel function of a small argument is used and analytically integrated. The integral expression proposed by Veysoglu et al [2] is also used to speed up the convergence of the evaluation of the Hankel function sum in the periodic Green's function. After solving the matrix equation to determine the surface fields, the coefficients of the reflected and transmitted Floquet modes can be calculated. If the reflected fields in region zero are written as

$$
\begin{aligned}
& E_{0 y}^{r}=\sum_{n} b_{n} e^{i k_{0 n}^{+} \cdot \bar{\rho}} \\
& H_{0 y}^{r}=\sum_{n} b_{n}^{(h)} e^{i K_{0 n}^{+} \cdot \bar{\rho}}
\end{aligned}
$$


and the transmitted fields in region two as

$$
\begin{aligned}
& E_{2 y}^{t}=\sum_{n} A_{n} e^{i k_{2 n}^{-} \cdot \bar{\rho}} \\
& H_{2 y}^{t}=\sum_{n} A_{n}^{(h)} e^{i k_{2 n}^{-} \cdot \bar{\rho}}
\end{aligned}
$$

the coefficients are given in terms of the surface fields by

$$
\begin{aligned}
& b_{n}=\frac{1}{2 i k_{0,} P} \int_{P(S 1)} d S\left[\frac{e^{-i k_{0 n}^{-} \cdot \bar{\rho}}}{\beta_{n}} \hat{n}_{1} \cdot \nabla, E_{0 y}(\bar{\rho})\right. \\
& \left.-E_{0 y}(\bar{\rho}) \hat{n}_{1} \cdot \nabla, \frac{e^{-i k_{0 n}^{-} \cdot \bar{\rho}}}{\beta_{n}}\right] \\
& b_{n}^{(h)}=\frac{1}{2 i k_{0 s} P} \int_{P(S 1)} d S\left[\frac{e^{-i k_{0 n}^{-} \cdot \bar{\rho}}}{\beta_{n}} \hat{n}_{1} \cdot \nabla, H_{0 y}(\bar{\rho})\right. \\
& \left.-H_{\mathrm{oy}_{y}}(\bar{\rho}) \hat{n}_{1} \cdot \nabla \cdot \frac{e^{-i \bar{k}_{0 n}^{-} \cdot \bar{\rho}}}{\beta_{n}}\right] \\
& A_{n}=\frac{1}{2 i k_{2 \varepsilon} P} \int_{P(S 2)} d S\left[\frac{e^{-i k_{2 n}^{-} \cdot \bar{\rho}}}{\beta_{n}} \hat{n}_{2} \cdot \nabla_{\bullet} E_{2 y}(\bar{\rho})\right. \\
& \left.-E_{2 y}(\bar{\rho}) \hat{n}_{2} \cdot \nabla \cdot \frac{e^{-i k_{2 n}^{-} \cdot \bar{\rho}}}{\beta_{n}}\right] \\
& A_{n}^{(h)}=\frac{1}{2 i k_{2 s} P} \int_{P(S 2)} d S\left[\frac{e^{-i k_{2 n}^{-} \cdot \bar{s}}}{\beta_{n}} \hat{n}_{2} \cdot \nabla \cdot H_{2 y}(\bar{\rho})\right. \\
& \left.-H_{2 y}(\bar{\rho}) \hat{n}_{2} \cdot \nabla \cdot \frac{e^{-i \bar{k}_{2 n}^{-} \cdot \bar{\rho}}}{\beta_{n}}\right]
\end{aligned}
$$

where $\bar{k}_{j n}^{-}$is defined as in the EBC section.

As with the EBC, once the mode amplitudes are determined, the emissivity can be found by integrating the total power reflected over the upper hemisphere and then applying Kirchhoff's Law. Note that the method of moments does not suffer from the problems of the EBC when the height-to-period ratio becomes too large. A larger number of basis functions is required and the calculations take longer to perform, but the method ultimately yields an accurate result. 


\subsection{Comparison of Methods}

Both the EBC and MOM methods were used to perform a theoretical analysis of the surface in the experiment. Figure 2 is a plot of the four polarimetric brightness temperatures calculated by both the EBC and MOM. Also shown in these plots are the corresponding brightness temperatures for a periodic water surface without the fiberglass layer on top. These calculations are for the experimental parameters described in the next section assuming a physical temperature of $300 \mathrm{~K}$. The EBC and MOM results are seen to agree, indicating that the surface slope limitations of the EBC are not being exceeded and that the numerical resolution of the method of moments code is adequate. The more efficient EBC method was used for the rest of the theoretical calculations presented. It is also seen that the effect of the layer is to increase $T_{B h}, T_{B v}$, and $T_{B p}$ by approximately $30 K$ over the single-layer brightnesses, but the effect on $U_{B}$ is small due to cancellation of this increase when the brightnesses in the three polarizations are used to compute $U_{B}$. Finally, the characteristic "jumps" in brightness temperature for an infinite periodic surface due to the transition of a Floquet mode from propagating to non-propagating are seen in the $T_{B h}$ curve. Note that the exact shape of the curve may not be the same as that of Figure 2 , as the curve was linearly interpolated between the calculated points every 15 degrees in azimuth.

\section{Experiment Setup}

To form the periodic water surface, a sinusoidal sheet of fiberglass $1.32 \mathrm{~m} \times 1.22 \mathrm{~m} \mathrm{x}$ $0.002 \mathrm{~m}$ thick was placed on top of a $1.83 \mathrm{~m}$ side square pool of fresh water of $0.23 \mathrm{~m}$ depth. The height and period of the sinusoidal corrugation were $1.4 \mathrm{~cm}$ peak-to-peak and $6.8 \mathrm{~cm}$ respectively. To prevent water from flowing over the edges, a styrofoam border approximately $3 \mathrm{~cm}$ high was glued around the surface.

The temperature of the water was measured around the surface in the experiment and found to be $22.5^{\circ} \pm 1.5^{\circ} \mathrm{C}$ throughout the experiment. As fresh water was used, this 
corresponds to an average dielectric constant of $62+i 32$ at $10 \mathrm{GHz}$ over this temperature range [7]. The dielectric constant of the fiberglass layer was measured using a network analyzer technique [6] from 12 to $18 \mathrm{GHz}$ and found to be $3.1 \pm 0.1+i(0.05 \pm 0.05)$ over this entire band. It was assumed that this dielectric constant was the same at $10 \mathrm{GHz}$.

The radiometer used in the experiment is the same as that used in [3]. It operates at $10 \mathrm{GHz}$ with a $200-\mathrm{MHz} \mathrm{RF}$ bandwidth, $-3 \mathrm{mV} / \mathrm{K}$ sensitivity, $\pm 1 \mathrm{~K}$ precision, and a pyramidal (standard gain) horn of $30^{\circ}$ beamwidth. The radiometer was mounted on a tripod at an elevation of $1.7 \mathrm{~m}$ above the pool surface and directed toward the surface along a direction determined by the azimuthal angle, $\phi$, and the polar angle, $\theta$ (see Figure 1). The radiometer was calibrated by viewing an absorber whose physical temperature was known for the hot load, and by viewing the sky at a set of angles for the cold load. Since the measured sky voltage can be expressed as a function of polar angle, the points measured can be extrapolated to obtain a radiometer voltage corresponding to brightness temperature $3 K$. This voltage is then used as the cold reference for the calibration.

Observations of the periodic surface were made as a function of azimuthal angle, which was varied by rotating the periodic surface in the pool so that the background noise contribution from the antenna sidelobes would remain constant through a set of measurements. The horn of this single polarization radiometer was rotated to produce measurements of $T_{B h}, T_{B v}$, and $T_{B p}$. Measurements of $T_{B r}$ were not possible with this radiometer; however, the $V_{B}$ brightness was not predicted to be significant.

\section{Estimation of Noise Contribution}

The contribution of background noise to the measured antenna temperature for the periodic surface can be large: noise from the antenna sidelobes and from reflections of sky brightness off the surface both contribute to error in the measurements. Since this background noise could not be easily eliminated from the experiment, estimates of the background contribution were made so that the noise could later be removed. The contribution of the 
background noise was estimated by measuring the brightness temperature of a flat water surface alone and by measuring the brightness temperature of a reflector in the pool which was the same size as the periodic surface. The theoretical results for both of these cases are known; thus, these measurements form a sort of two-point calibration for the periodic surface when it is in the pool.

Consider the case of the observation of the water in the pool alone. The measured brightness temperature, $T_{\text {flat } M}$, consists of contributions from the water surface, $T_{\text {flat }}$, from the ground beside the pool and the sides of the pool, $T_{\text {side, }}$ and from reflections of the sky temperature, $T_{\text {sky }}$, off the pool. The contribution of the reflections of the sky temperature from the ground beside the pool is neglected. If we assume that there is no variation in these temperatures with the incidence angle, then the relationship between these variables can be written as:

$$
T_{\text {flat } M}=T_{\text {flat }}\left(1-f_{\bullet}\right)+T_{\text {sky }}\left(1-f_{\bullet}\right)\left(1-T_{\text {flat }} / T_{P_{\text {wat }}}\right)+T_{\text {side }} f_{\text {s }}
$$

where $f_{\text {s }}$ represents the fraction of the antenna pattern that does not view the pool and $T_{P_{w a t}}$ is the physical temperature of the water in the pool. Measured values of $f_{0}$ ranged from 3 to 18 percent through the experiment. Both $T_{\text {sky }}$ and $T_{\text {side }}$ were obtained from the experiment at angles corresponding to the specular reflection for the sky temperature and direct incidence for the ground temperature.

Next consider the case of a reflector in the pool the same size and at the same location as the periodic surface. The brightness temperature measured, $T_{\text {reflM }}$, consists of the reflection of the sky temperature over the reflector surface, the flat water surface temperature over the remainder of the pool and the reflection of the sky temperature from this portion of the surface, and the ground contribution. This can be expressed as:

$$
\begin{aligned}
T_{r e f l M}= & T_{\text {sky }} f_{r}+T_{\text {sky }}\left(1-f_{r}-f_{\imath}\right)\left(1-T_{\text {flat }} / T_{P \text { wat }}\right) \\
& +T_{\text {flat }}\left(1-f_{r}-f_{s}\right)+T_{\text {side }} f_{\iota} \\
= & T_{f l a t M}+T_{\text {flat }} f_{r}\left(T_{\text {sky }} / T_{P \text { wat }}-1\right)
\end{aligned}
$$


where $f_{r}$ is the fraction of the antenna pattern that views the reflector surface, which was found to range from 50 to 74 percent through the experiment. A further assumption that the physical temperature of the water and the background sky temperature are the same in the flat surface and reflector measurements is made in the second equality.

Finally, consider the case of the periodic surface in the pool. The measured temperature, $T_{\text {sur } f M}$, consists of contribution from the periodic surface, the flat water in the pool, sky reflections off the pool area, and the side contribution. The reflections of sky temperature off the periodic surface are determined by its Floquet modes; however, since the sky temperature is assumed uniform, this reduces to the same case as that of a specular reflector. The measured temperature can be expressed as:

$$
\begin{aligned}
& T_{\text {sur } f M}=T_{\text {surf } f} f_{r}+T_{\text {sky }} f_{r}\left(1-T_{\text {our } f} / T_{P_{w a t}}\right)+T_{f l a t}\left(1-f_{r}-f_{s}\right) \\
& +T_{\text {sky }}\left(1-T_{f l a t} / T_{P \text { wat }}\right)\left(1-f_{r}-f_{s}\right)+T_{\text {side }} f_{\text {s }} \\
& =T_{\text {reflM }}+T_{\text {surf } f} f_{r}\left(1-T_{\text {sky }} / T_{P_{\text {wat }}}\right)
\end{aligned}
$$

These three equations can be used to solve for $T_{\text {surf }}$, the periodic surface brightness temperature averaged over the fraction of the antenna pattern on the surface:

$$
T_{\text {surf }}=\frac{T_{\text {flat }}}{T_{\text {flat } M}-T_{\text {refl }}}\left(T_{\text {sur } f M}-T_{\text {reflM }}\right)
$$

This brightness temperature has the effects of both the reflections of the sky temperature off the surface and the antenna field of view outside of the periodic surface removed.

This calibration procedure increases the effect of the radiometer measurement uncertainty in the final calculated $T_{\text {surf }}$ to approximately $3 \mathrm{~K}$. The assumptions made in the above equations also contribute to the error in the experiment. A theoretical study in which the brightness contributions of the water around the surface and the sky temperature were estimated by integrating the angularly varying $T_{\text {flat }}$ and $T_{\text {sky }}$ over a theoretical antenna pattern indicated that the use of the specular $T_{f l a t}$ and $T_{s k y}$ values were valid to within $2 \mathrm{~K}$. A bias in both of these values would produce a systematic error of less than $5 \mathrm{~K}$. One remaining possible source of error is the possibility of the close-to-the-horizon 
Floquet modes of the periodic surface imaging something with a higher brightness temperature than the sky. Note that the $U_{B}$ parameter is fairly insensitive to any systematic error in the calibration used. Since systematic error will tend to affect all three polarizations in a similar manner, the effects tend to cancel out, leaving $U_{B}$ unaffected.

The results presented in Section 6 for the measurements of periodic surface brightness temperatures were obtained using the above technique.

\section{Experimental Results}

Table 1 presents the measurement results at $\theta=20^{\circ}$. Shown are the measured emissivities $e_{h}, e_{v}$, and $e_{p}$. The emissivity of the third Stokes brightness parameter is then obtained as $e_{U}=e_{h}+e_{v}-2 e_{p}$. This formula is opposite in sign to that previously given due to the fact that the radiometer polarization basis was actually rotated to $-45^{\circ}$ in the experiment. It is observed from the experiment that, in general, the $e_{U}$ value approaches a maximum absolute value at $\phi=-45^{\circ}$ and approaches zero at $\phi=-90^{\circ}$ and $\phi=0^{\circ}$. This shows that the $U_{B}$ parameter is sensitive to the azimuthal direction of a periodic water surface. These trends are also seen in Table 2 , which presents the measurement results at $\theta=30^{\circ}$.

\section{Comparison with Theory}

Figures 2 and 3 are plots of the measurement results and the theoretically predicted brightness temperatures for polar angles $20^{\circ}$ and $30^{\circ}$, respectively, normalized to a constant physical temperature of $22.5 \mathrm{C}$. The theoretical values plotted were obtained using the two-layer version of the Extended Boundary Condition method. These theoretical results were also averaged noncoherently over 81 points in a three-dimensional calculated antenna pattern for the pyramidal horn used in the experiment, which tends to smooth out the abrupt changes obtained for plane wave incidence in section 3 . This pattern was obtained from [8], using the measured antenna dimensions of $5.7 \mathrm{~cm}$ by $7 \mathrm{~cm}$ for the aperture dimension and $10 \mathrm{~cm}$ and $8.9 \mathrm{~cm}$ for the corresponding flare lengths of the horn. Good 
general agreement is observed between the theory and experiment, indicating that the background noise removal procedure was reasonable. However, there is a bias of the experimental results above the theoretical predictions of approximately $10 \mathrm{~K}$ for $T_{B h}, T_{B v}$ and $T_{B_{P}}$ and $6 \mathrm{~K}$ for $U_{B}$. One possible source of this error is the permittivity value for the fiberglass surface used in the experiment, which could be slightly higher at $10 \mathrm{GHz}$ than the measured value at $12.4 \mathrm{GHz}$. A increase in this permittivity to $3.2+i 0.1$ increases the theoretical predictions by approximately $5 \mathrm{~K}$ or 0.017 emissivity. A second possible source of the error is the use of the specular values for $T_{\text {flat }}$ and $T_{a k y}$ as discussed in section 5 . This could also contribute approximately $5 \mathrm{~K}$ or 0.017 emissivity variation. Finally, the imaging of a brightness source other than the sky by the Floquet modes of the periodic surface would tend to increase the experimental brightness temperatures above the theoretical predictions. However, the area in which the experiment was performed was clear to about ten degrees above the horizon. Only the azimuthal angles $-90,-15,0$, and 15 are theoretically predicted to have modes this close to the horizon.

\section{Conclusion}

We have demonstrated in this paper that the $U_{B}$ parameter exists for a two-layer periodic water surface and can approach brightnesses exceeding $30 \mathrm{~K}$ at $X$ band. We have also shown that the $U_{B}$ parameter is fairly insensitive to the effects of the fiberglass layer and the measurement uncertainties in the experiment as an indicator of surface azimuthal direction. Although the periodic water surface is an extremely simplified model of an actual ocean surface, this experiment further strengthens the idea of using passive polarimetry to infer wind direction over the ocean. Further research into this area and into other applications of passive polarimetric remote sensing will continue. 
Acknowledgements This work was supported in part by ONR Grant N00014-92-J1616, NASA grant NAGW-1617, and a National Science Foundation Graduate Research Fellowship. The authors would like to thank M. A. Tassoudji of MIT for assistance with surface construction and M. E. Veysoglu of MIT and S. H. Yueh of JPL for helpful discussions on the theoretical formulation. 


\section{REFERENCES}

[1] Tsang, L., "Polarimetric passive microwave remote sensing of random discrete scatterers and rough surfaces," J. Electromag. Waves Applic., Vol. 5, No. 1, 41-57, 1991.

[2] Veysoglu, M. E., H. A. Yueh, R. T. Shin and J. A. Kong, "Polarimetric passive remote sensing of periodic surfaces," J. Electromag. Waves Applic., Vol. 5, No. 3, 267-280, 1991.

[3] Nghiem, S. V., M. E. Veysoglu, J. A. Kong, R. T. Shin, K. O'Neill, and A. W. Lohanick, "Polarimetric passive remote sensing of a periodic soil surface: microwave measurements and analysis," J. Electromag. Waves Applic., Vol. 5, No. 9, 997-1005, 1991.

[4] Tsang, L., J. A. Kong, and R. T. Shin, Theory of Microwave Remote Sensing, John Wiley, New York, 1985.

[5] Chuang, S. L. and J. A. Kong, "Wave scattering from a periodic dielectric surface for a general angle of incidence," Radio Science, Vol. 17, No. 3, 545-557, 1982.

[6] Hewlett Packard Co., "Measuring the dielectric constant of solids with the HP 8510 network analyzer," Product Note 8510-3.

[7] Klein, L. A. and C. T. Swift, "An improved model for the dielectric constant of sea water at microwave frequencies," IEEE Trans. Ant. Propag., AP-25, 104-111, 1977.

[8] Jull, E. V., Aperture Antennas and Diffraction Theory, P. Peregrinus, New York, 55-60, 1981. 
[9] Harrington, R. F., Field Computation by Moment Methods, Macmillan, New York, 1968.

[10] Garcia, N., V. Celli, N. Hill, and N. Cabrera, "Ill conditioned matrices in the scattering of waves from hard corrugated surfaces," Physical Review B, Vol. 18, No. 10, 5184-89, 1978. 


\section{Appendix A: EBC Coupled Matrix Equation}

The matrix equation of the $\mathrm{EBC}$ can be written as:

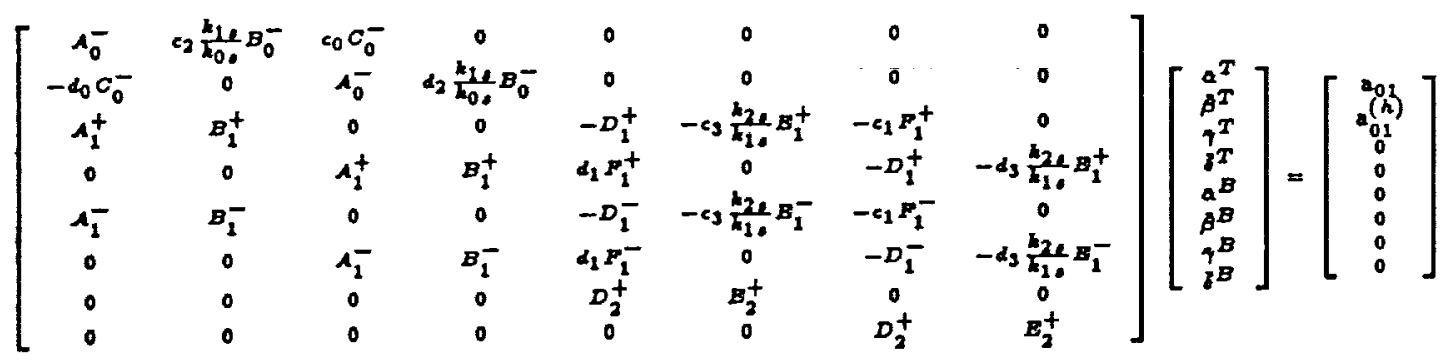

where

$$
\begin{aligned}
& {\left[A_{j}^{ \pm}\right]_{m n}=\frac{1}{i k_{j,} P} \int_{P(S 1)} d \sigma \hat{n}_{1} \cdot \nabla \cdot \frac{e^{-i k_{j m}^{ \pm} \cdot \hat{\rho}_{0}(x)}}{\sqrt{\beta_{j m}}} \exp \left[i k_{x i} x+i \frac{2 n \pi}{P} x\right]} \\
& =\frac{-1+\alpha_{j m} \alpha_{j n}}{P\left( \pm \beta_{j m}\right) \sqrt{\beta_{j m}}} \int_{P(S 1)} d x \exp \left[-i(m-n) \frac{2 \pi}{P} x-i k_{j \boldsymbol{s}}\left( \pm \beta_{j m}\right) f_{1}(x)\right] \\
& {\left[B_{j}^{ \pm}\right]_{m n}=\frac{-1}{P} \int_{P(S 1)} d x \frac{e^{-i k_{j m}^{ \pm} \cdot \bar{\rho}_{o}(x)}}{\sqrt{\beta_{j m}}} \exp \left[i k_{x \mathrm{i}} x+i \frac{2 n \pi}{P} x\right]} \\
& =\frac{-1}{P \sqrt{\beta_{j m}}} \int_{P(S 1)} d x \exp \left[-i(m-n) \frac{2 \pi}{P} x-i k_{j\lrcorner}\left( \pm \beta_{j m}\right) f_{1}(x)\right] \\
& {\left[C_{j}^{ \pm}\right]_{m n}=\alpha_{j n}\left[B_{j}^{ \pm}\right]_{m n}} \\
& {\left[D_{j}^{ \pm}\right]_{m n}=\frac{1}{i k_{j,} P} \int_{P(S 2)} d \sigma \hat{n}_{2} \cdot \nabla, \frac{e^{-i k_{j m}^{ \pm} \cdot \bar{p}_{\theta}(x)}}{\sqrt{\beta_{j m}}} \exp \left[i k_{x i} x+i \frac{2 n \pi}{P} x\right]} \\
& =\frac{-1+\alpha_{j m} \alpha_{j n}}{P\left( \pm \beta_{j m}\right) \sqrt{\beta_{j m}}} \int_{P(S 2)} d x \exp \left[-i(m-n) \frac{2 \pi}{P} x-i k_{j,}\left( \pm \beta_{j m}\right) f_{2}(x)\right] \\
& {\left[E_{j}^{ \pm}\right]_{m n}=\frac{-1}{P} \int_{P(S 2)} d x \frac{e^{-i k_{j m}^{ \pm} \cdot \bar{p}_{\Delta}(x)}}{\sqrt{\beta_{j m}}} \exp \left[i k_{x i} x+i \frac{2 n \pi}{P} x\right]} \\
& =\frac{-1}{P \sqrt{\beta_{j m}}} \int_{P(S 2)} d x \exp \left[-i(m-n) \frac{2 \pi}{P} x-i k_{j \Omega}\left( \pm \beta_{j m}\right) f_{2}(x)\right]
\end{aligned}
$$




$$
\left[F_{j}^{ \pm}\right]_{m n}=\alpha_{j n}\left[F_{j}^{ \pm}\right]_{m n}
$$

where $(S j)$ indicates that the integration is over surface $j$,

$$
a_{01 n}=\delta_{n 0} \sqrt{\beta_{0}} E_{0}\left(\hat{e}_{i} \cdot \hat{y}\right)
$$

and

$$
a_{01 n}^{(h)}=\delta_{n 0} \sqrt{\beta_{0}} \frac{E_{0}}{\omega \mu}\left(\bar{k}_{i} \times \hat{e}_{i} \cdot \hat{y}\right)
$$

The above $\vec{a}_{01}$ elements are determined by the $\hat{y}$ component of the incident electric and magnetic fields. Truncating the Fourier series of the surface field unknowns results in a finite matrix which can be inverted to obtain the surface field unknowns.

For the special case of two surfaces described by

$$
\begin{aligned}
& f_{1}(x)=-h_{1} \cos \left(\frac{2 \pi x}{P}\right) \\
& f_{2}(x)=-h_{2} \cos \left(\frac{2 \pi x}{P}\right)-d
\end{aligned}
$$

the matrix elements can be integrated and expressed in terms of Bessel functions as follows

$$
\begin{aligned}
& {\left[A_{j}^{ \pm}\right]_{m n}=\frac{-1+\alpha_{j m} \alpha_{j n}}{ \pm \beta_{j m} \sqrt{\beta_{j m}}}( \pm i)^{|m-n|} J_{|m-n|}\left(k_{j,}, h_{1} \beta_{j m}\right)} \\
& {\left[B_{j}^{ \pm}\right]_{m n}=\frac{-1}{\sqrt{\beta_{j m}}}( \pm i)^{|m-n|} J_{|m-n|}\left(k_{j,} h_{1} \beta_{j m}\right)} \\
& {\left[C_{j}^{ \pm}\right]_{m n}=\alpha_{j n}\left[B_{j}^{ \pm}\right]_{m n}} \\
& {\left[D_{j}^{ \pm}\right]_{m n}=\frac{-1+\alpha_{j m} \alpha_{j n}}{ \pm \beta_{j m} \sqrt{\beta_{j m}}}( \pm i)^{|m-n|} J_{|m-n|}\left(k_{j,} h_{2} \beta_{j m}\right) e^{ \pm i k_{j}, \beta_{j m} d}} \\
& {\left[E_{j}^{ \pm}\right]_{m n}=\frac{-1}{\sqrt{\beta_{j m}}}( \pm i)^{|m-n|} J_{|m-n|}\left(k_{j,} h_{2} \beta_{j m}\right) e^{ \pm i k_{j}, \beta_{j m} d}} \\
& {\left[F_{j}^{ \pm}\right]_{m n}=\alpha_{j n}\left[E_{j}^{ \pm}\right]_{m n}}
\end{aligned}
$$

where $J_{|m-n|}$ denotes the Bessel function. These Bessel functions were evaluated using a series form, which was summed until convergence to within a fractional accuracy of $10^{-6}$ was observed. 
Appendix B: MoM Matrix Equation

The matrix equation for the Method of Moments can be written as:

$$
\left[\begin{array}{cccccccc}
\overline{\bar{A}} & c_{2} \overline{\bar{B}} & c_{0} \overline{\bar{C}} & 0 & 0 & 0 & 0 & 0 \\
-d_{0} \overline{\bar{C}} & 0 & \overline{\bar{A}} & d_{2} \overline{\bar{B}} & 0 & 0 & 0 & 0 \\
\overline{\bar{D}} & \overline{\bar{E}} & 0 & 0 & \overline{\bar{F}} & c_{3} \overline{\bar{G}} & c_{1} \overline{\bar{H}} & 0 \\
0 & 0 & \overline{\bar{D}} & \overline{\bar{E}} & -d_{1} \overline{\bar{H}} & 0 & \overline{\bar{F}} & d_{3} \overline{\bar{G}} \\
\overline{\bar{I}} & \overline{\bar{J}} & 0 & 0 & \overline{\bar{K}} & c_{3} \overline{\bar{L}} & c_{1} \overline{\bar{M}} & 0 \\
0 & 0 & \overline{\bar{I}} & \overline{\bar{J}} & -d_{1} \overline{\bar{M}} & 0 & \overline{\bar{K}} & d_{3} \overline{\bar{L}} \\
0 & 0 & 0 & 0 & \overline{\bar{N}} & \overline{\bar{O}} & 0 & 0 \\
0 & 0 & 0 & 0 & 0 & 0 & \overline{\bar{N}} & \overline{\bar{O}}
\end{array}\right]\left[\begin{array}{c}
\bar{a} \\
\bar{b} \\
\bar{c} \\
\bar{d} \\
\bar{e} \\
\bar{f} \\
\bar{g} \\
\bar{h}
\end{array}\right]=\left[\begin{array}{c}
\bar{E}_{v i} \\
\bar{H}_{y i} \\
0 \\
0 \\
0 \\
0 \\
0 \\
0
\end{array}\right]
$$

where

$$
\begin{aligned}
& {[\bar{A}]_{m n}=\frac{\delta_{m n}}{2}+\int_{x_{n-1}(S 1)}^{x_{n}} \hat{n}_{1} \cdot \nabla, G_{0 P}\left(t_{m}, x\right) d S} \\
& {[\bar{B}]_{m n}=\int_{x_{n-1}(S 1)}^{x_{n}} G_{0 P}\left(t_{m}, x\right) d x} \\
& {[\bar{C}]_{m n}=G_{0 P}\left(t_{m}, x_{n}\right)-G_{0 P}\left(t_{m}, x_{n-1}\right)} \\
& {[\bar{D}]_{m n}=\frac{\delta_{m n}}{2}-\int_{x_{n-1}(S 1)}^{x_{n}} \hat{n}_{1} \cdot \nabla \cdot G_{1 P}\left(t_{m}, x\right) d S} \\
& {[\bar{E}]_{m n}=-\int_{x_{n-1}(S 1)}^{x_{n}} G_{1 P}\left(t_{m}, x\right) d x}
\end{aligned}
$$

with the testing points and integration on surface one,

$$
\begin{aligned}
& {[F]_{m n}=\int_{x_{n-1}(S 2)}^{x_{n}} \hat{n}_{1} \cdot \nabla, G_{1 P}\left(t_{m}, x\right) d S} \\
& {[\bar{G}]_{m n}=\int_{x_{n-1}(S 2)}^{x_{n}} G_{1 P}\left(t_{m}, x\right) d x} \\
& {[\bar{H}]_{m n}=G_{1 P}\left(t_{m}, x_{n}\right)-G_{1 P}\left(t_{m}, x_{n-1}\right)}
\end{aligned}
$$

with the testing points on surface one, integration on surface two,

$$
\begin{aligned}
& {[\bar{I}]_{m n}=-\int_{x_{n-1}(S 1)}^{x_{n}} \hat{n}_{1} \cdot \nabla_{\cdot} G_{1 P}\left(t_{m}, x\right) d S} \\
& {[\bar{J}]_{m n}=-\int_{x_{n-1}(S 1)}^{x_{n}} G_{1 P}\left(t_{m}, x\right) d x}
\end{aligned}
$$

with the testing points on surface two, integration on surface one, and

$$
[\bar{K}]_{m n}=\frac{\delta_{m n}}{2}+\int_{x_{n-1}(S 2)}^{x_{n}} \hat{n}_{1} \cdot \nabla, G_{1 P}\left(t_{m}, x\right) d S
$$




$$
\begin{aligned}
& {[\bar{L}]_{m n}=\int_{x_{n-1}(S 2)}^{x_{n}} G_{1 P}\left(t_{m}, x\right) d x} \\
& {[\bar{M}]_{m n}=G_{1 P}\left(t_{m}, x_{n}\right)-G_{1 P}\left(t_{m}, x_{n-1}\right)} \\
& {[\bar{N}]_{m n}=\frac{\delta_{m n}}{2}-\int_{x_{n-1}(S 2)}^{x_{n}} \hat{n}_{1} \cdot \nabla, G_{2 P}\left(t_{m}, x\right) d S} \\
& {[\bar{O}]_{m n}=\int_{x_{n-1}(S 2)}^{x_{n}} G_{2 P}\left(t_{m}, x\right) d x}
\end{aligned}
$$

with the testing points and integration on surface two. The above integrals were approximated by a single point rectangular area at the testing point. This approximation becomes more accurate as the number of testing points is increased. In the results presented, 150 basis functions were used, which corresponds to 66 basis functions per wavelength in the free space region. This large number of basis functions was needed due to the much shorter wavelength in the high permittivity water region. 
Table 1: Results of measurements at $X$ band for $\theta=20^{\circ}$

\begin{tabular}{|c||l|l|l|l|}
\hline$\phi\left(^{\circ}\right)$ & $e_{h}$ & $e_{v}$ & $e_{p}$ & $e_{U}$ \\
\hline \hline-120 & .561 & .530 & .591 & -.091 \\
\hline-105 & .595 & .527 & .591 & -.060 \\
\hline-90 & .611 & .500 & .554 & .003 \\
\hline-75 & .581 & .497 & .503 & .072 \\
\hline-60 & .564 & .530 & .500 & .094 \\
\hline-45 & .486 & .503 & .429 & .131 \\
\hline-30 & .466 & .561 & .452 & .123 \\
\hline-15 & .470 & .605 & .497 & .081 \\
\hline 0 & .466 & .632 & .530 & .038 \\
\hline 15 & .490 & .635 & .568 & -.011 \\
\hline 30 & .497 & .595 & .578 & -.064 \\
\hline
\end{tabular}

Table 2: Results of measurements at $X$ band for $\theta=30^{\circ}$

\begin{tabular}{|c||l|l|l|c|}
\hline$\phi\left(^{\circ}\right)$ & $e_{h}$ & $e_{v}$ & $e_{p}$ & $U$ \\
\hline \hline-120 & .524 & .541 & .584 & -.103 \\
\hline-105 & .557 & .534 & .578 & -.065 \\
\hline-90 & .564 & .510 & .557 & -.040 \\
\hline-75 & .527 & .500 & .493 & .041 \\
\hline-60 & .527 & .530 & .493 & .071 \\
\hline-45 & .446 & .510 & .420 & .116 \\
\hline-30 & .449 & .568 & .453 & .111 \\
\hline-15 & .453 & .622 & .514 & .047 \\
\hline 0 & .463 & .632 & .544 & .007 \\
\hline 15 & .486 & .649 & .574 & -.013 \\
\hline 30 & .497 & .618 & .581 & -.047 \\
\hline
\end{tabular}




\section{$9 \quad$ Figure Captions}

$1+4$

Figure 1: Geometry of a "two-layer" periodic surface

Figure 2: Comparison of EBC and MOM brightness temperatures at polar angle $20^{\circ}$ versus azimuthal angle (a) $T_{B h}$ (b) $T_{B v}$ (c) $U_{B}$ (d) $V_{B}$

Figure 3: Comparison of theoretical and experimental brightness temperatures at polar angle $20^{\circ}$ versus azimuthal angle (a) $T_{B h}$ (b) $T_{B v}$ (c) $U_{B}$ (d) $T_{B_{p}}$

Figure 4: Comparison of theoretical and experimental brightness temperatures at polar angle $30^{\circ}$ versus azimuthal angle (a) $T_{B h}$ (b) $T_{B v}$ (c) $U_{B}$ (d) $T_{B_{p}}$ 


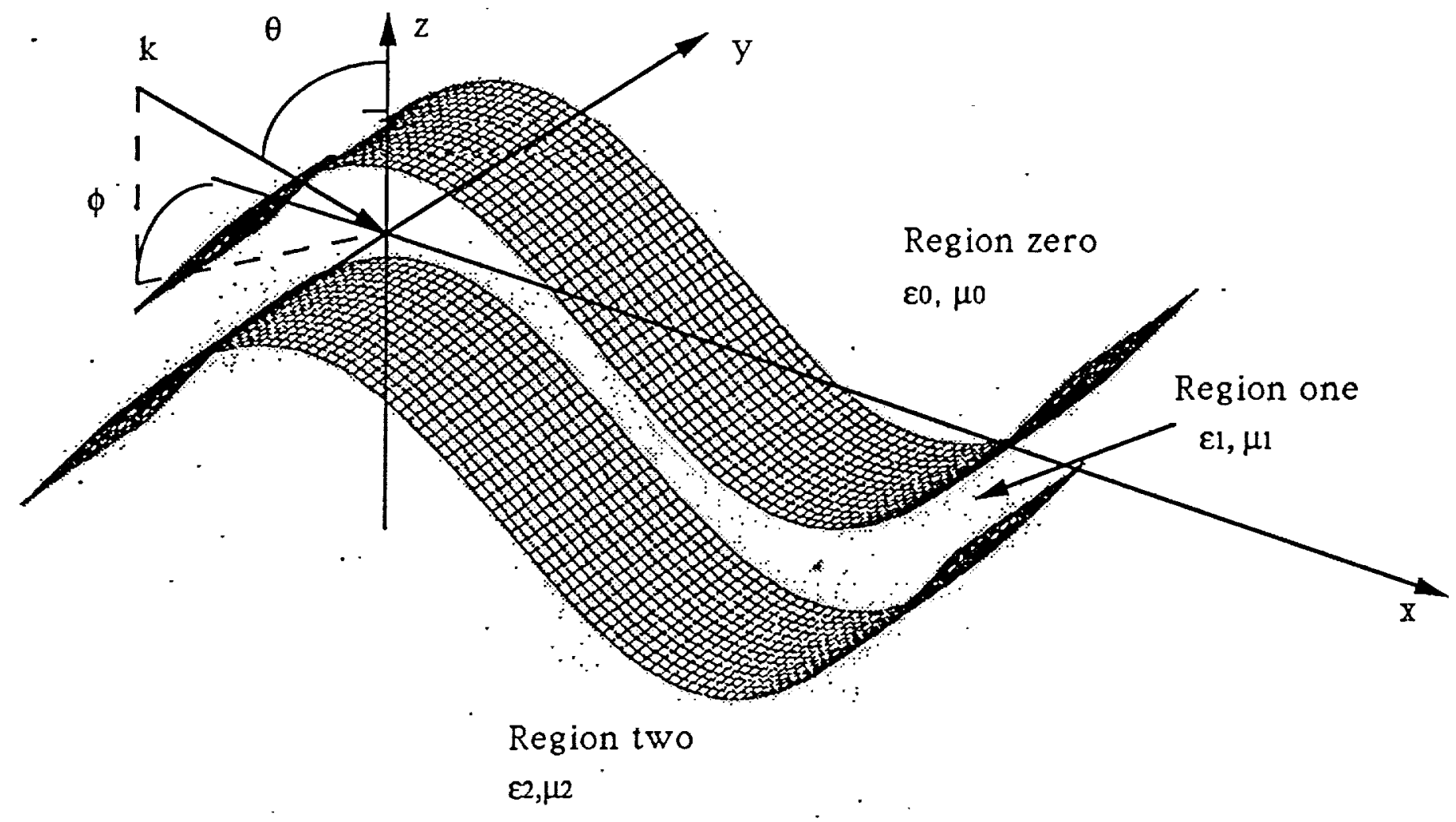

32- Figure One (Johnso; etal) 
$\because$

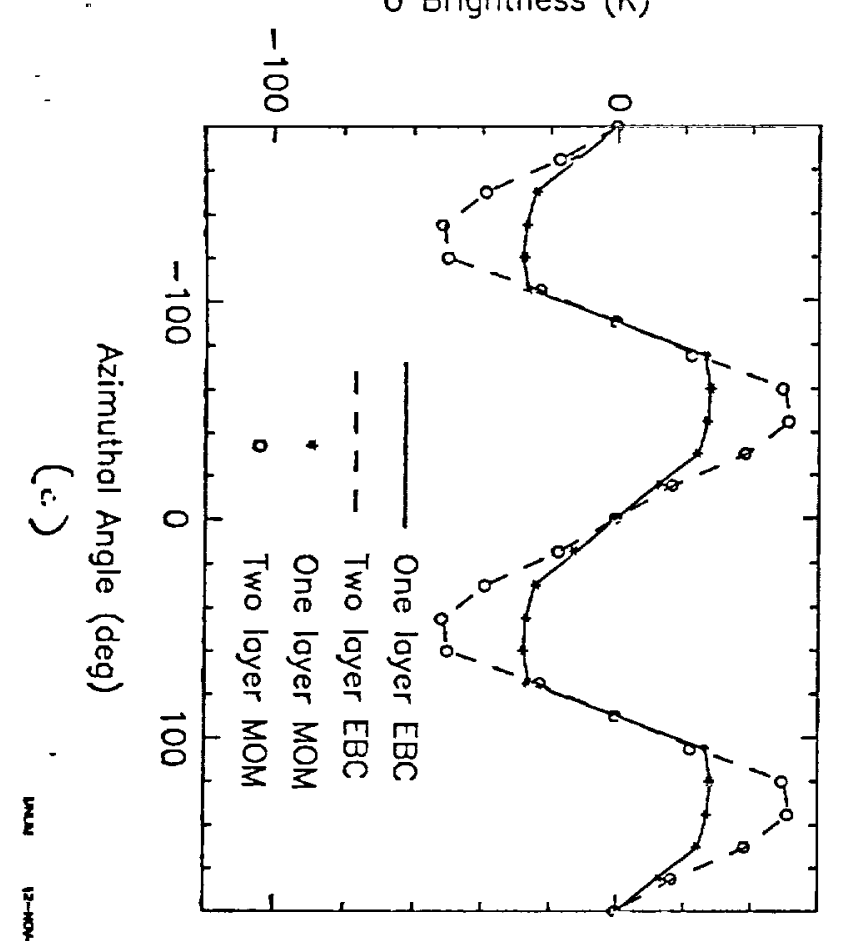

U Brightness (K)

$\frac{5}{2}$
Horizontal Brightness (K)

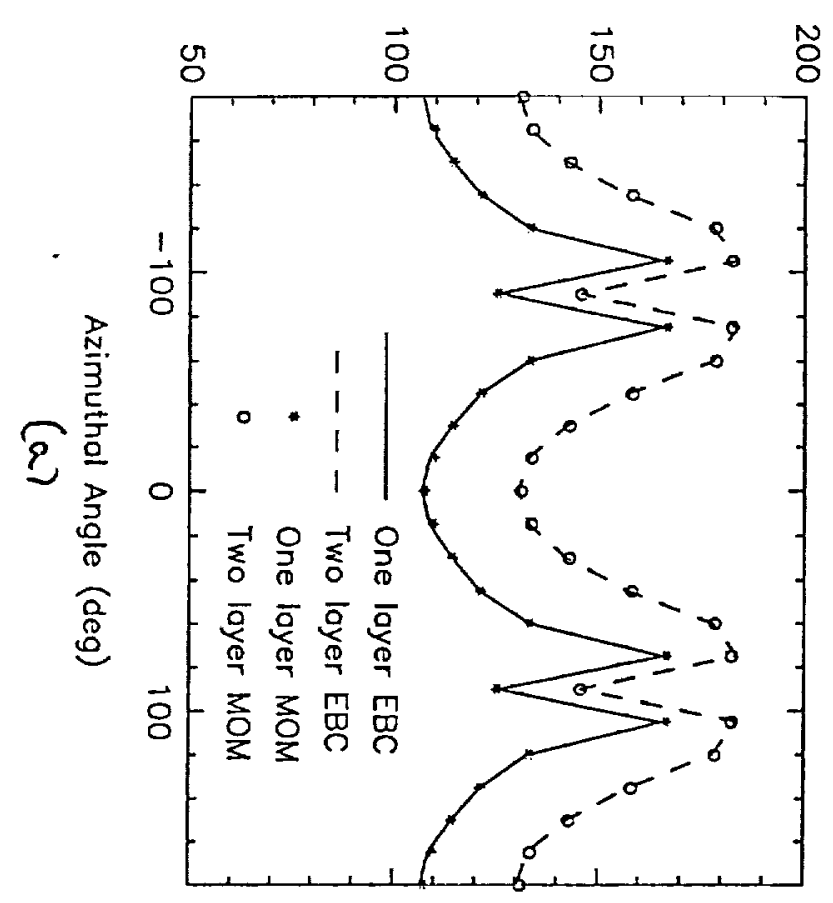

Vertical Brightness (K)

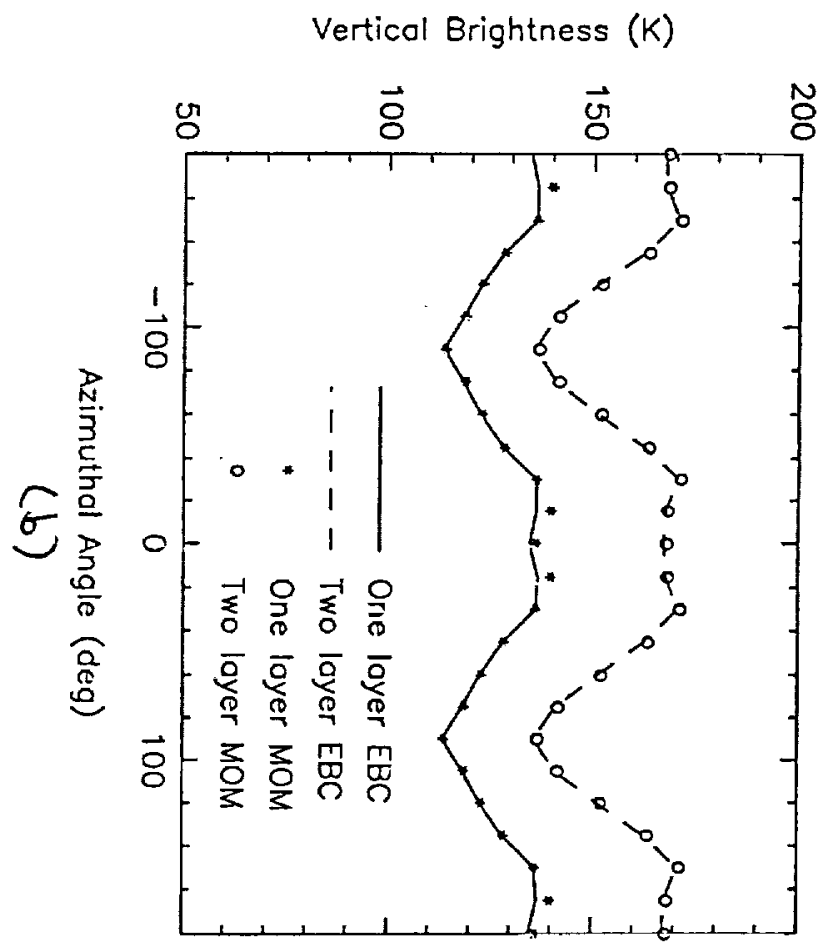

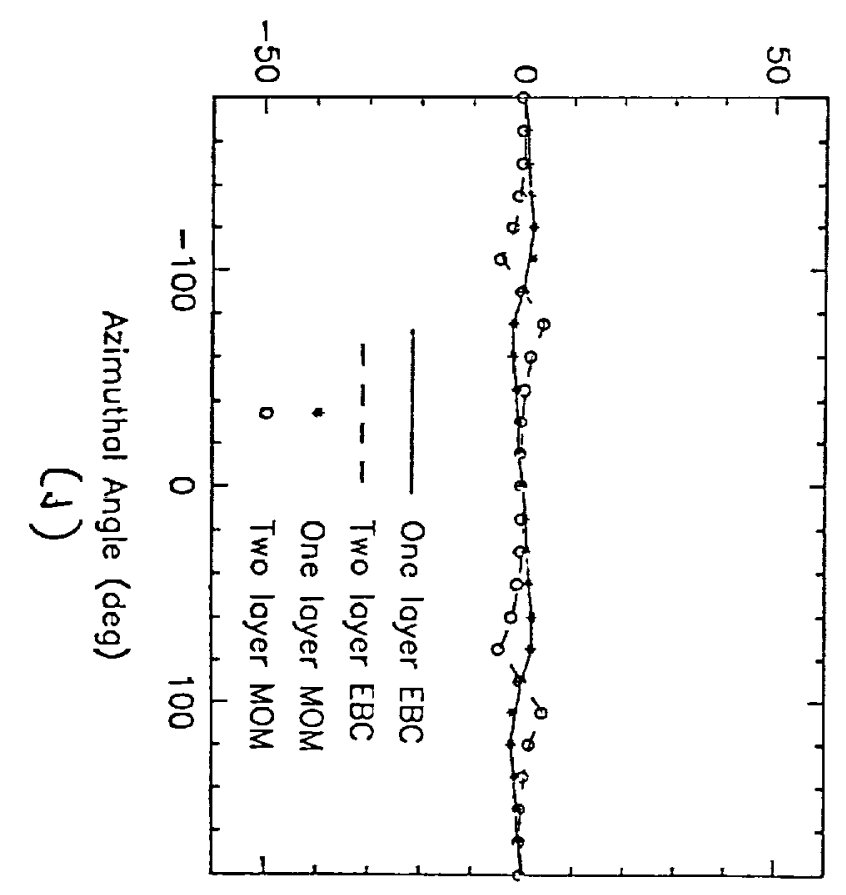

33. Figure twe (Jihnser at al) 

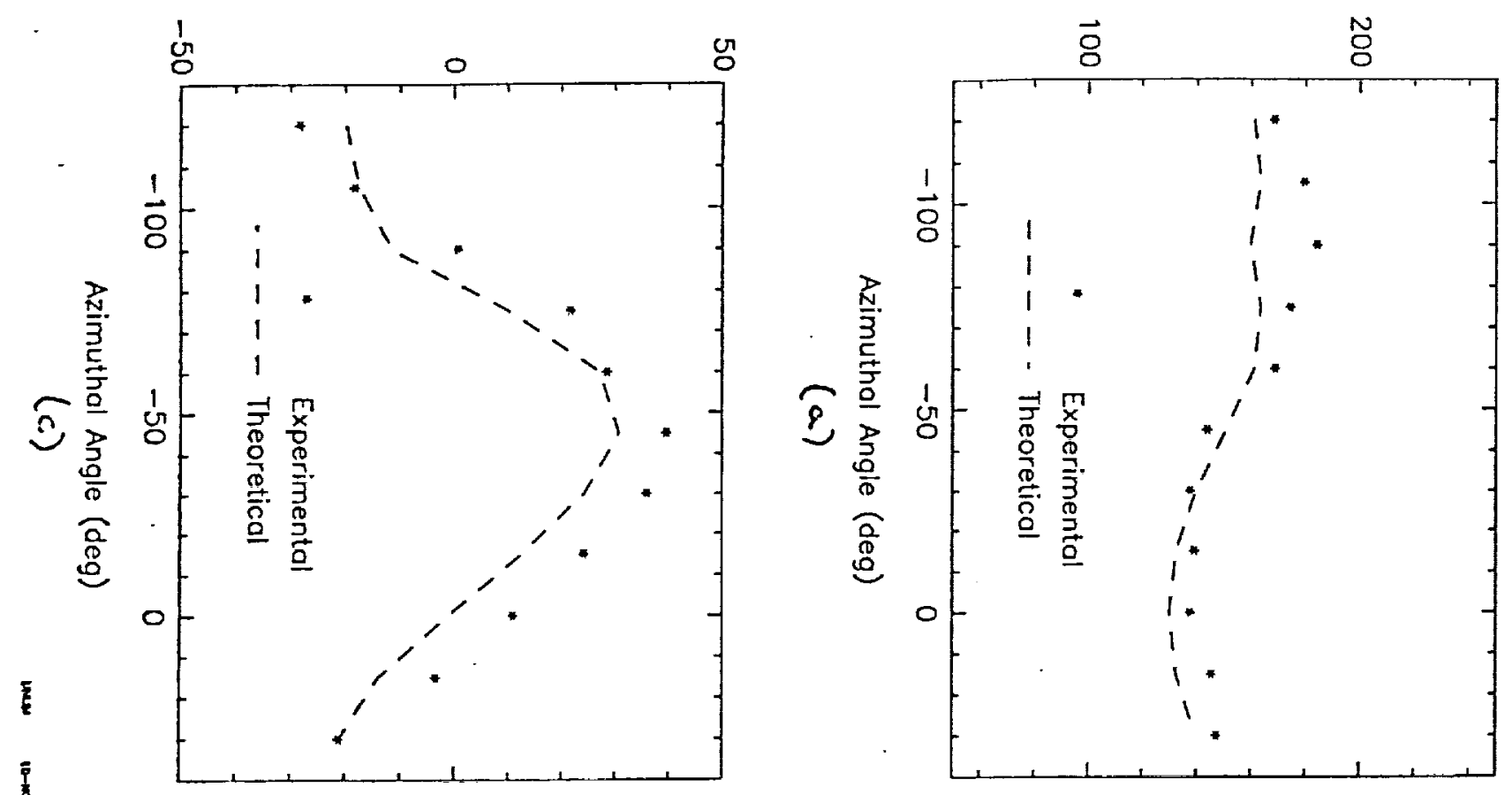

$\frac{1}{5}$
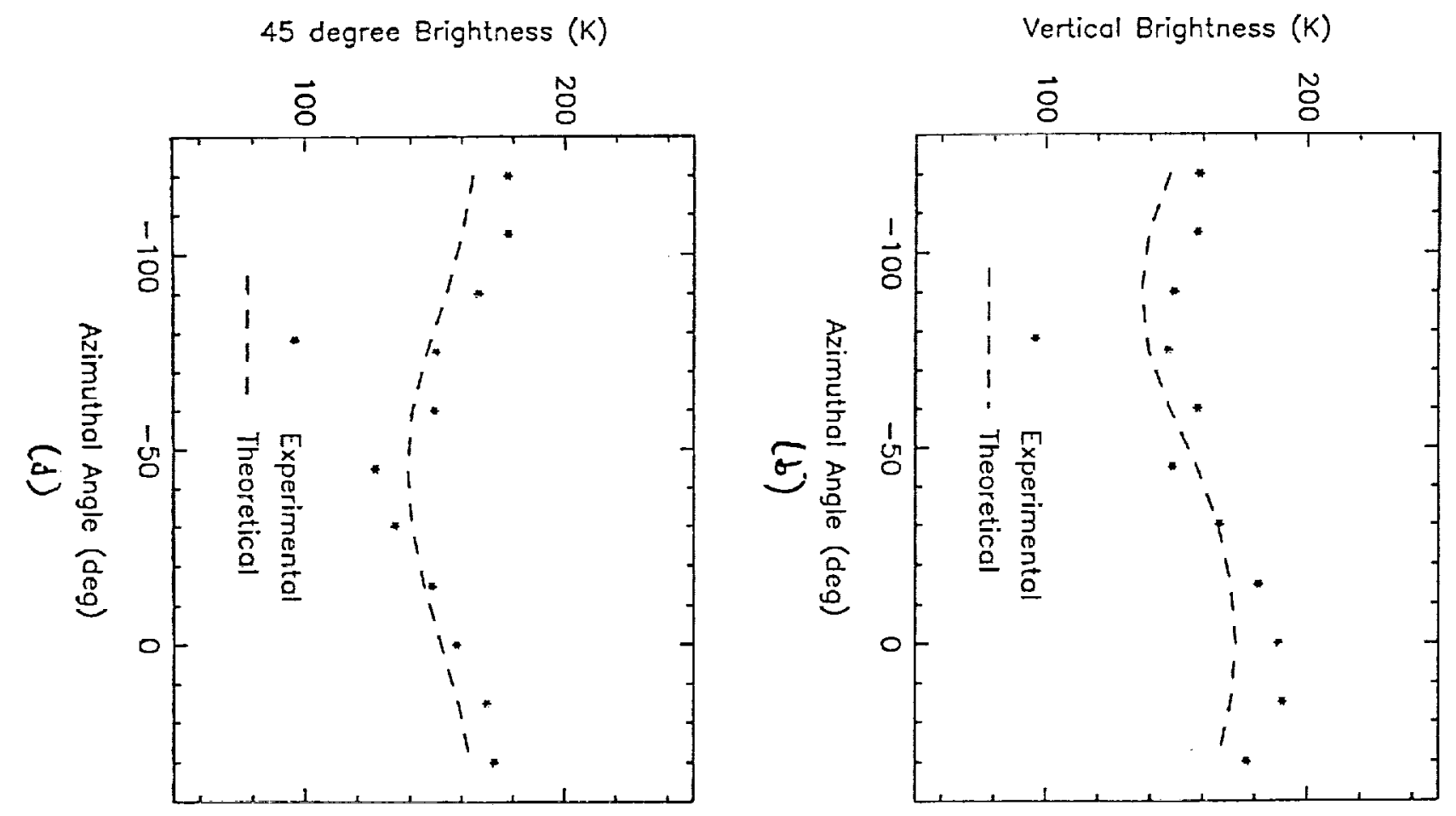

34-Figure 3 (Jotinsucetal) 

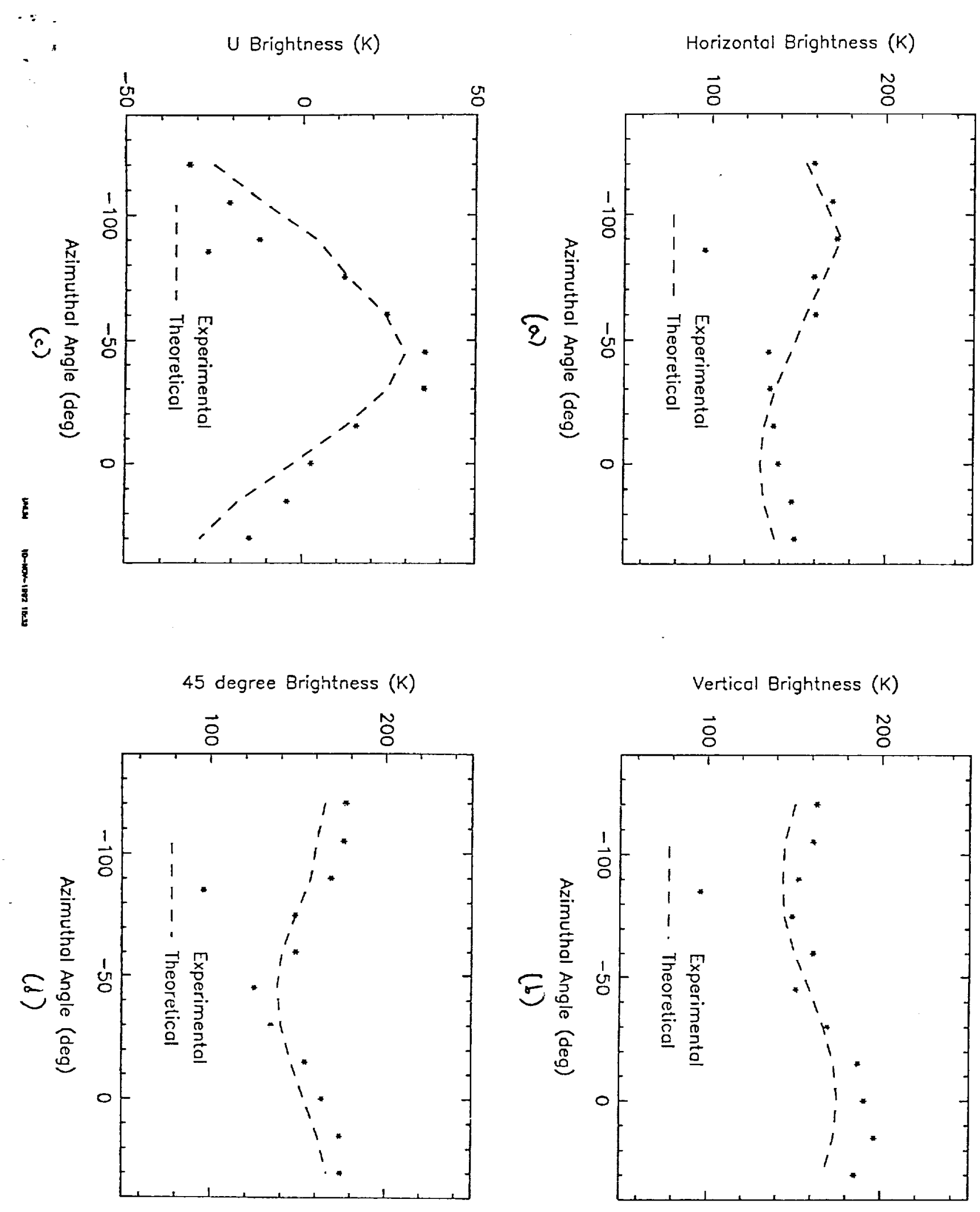

$35-F_{\text {igure }} 4$ (Johnsometal) 
- 